\title{
VARIACION LATITUDINAL Y ESTACIONAL EN LADIETADE DISSOSTICHUS ELEGINOIDES SMITT, 1898 (PERCIFORMES: NOTOTHENIIDAE) EN AMBIENTES PROFUNDOS DE LA COSTA CENTRO-SUR DE CHILE
}

\author{
LATITUDINAL AND TEMPORAL VARIATION IN THE DIET OF DISSOSTICHUS \\ ELEGINOIDES SMITT, 1898 (PERCIFORMES: NOTOTHENIIDAE) IN DEEP \\ ENVIRONMENTS OF THE SOUTH AND CENTER COAST OF CHILE
}

\author{
Carlos Murillo $^{1}$, Ciro Oyarzún ${ }^{2} \&$ Italo Fernández ${ }^{1}$ \\ ${ }^{1}$ Programa de Graduados en Zoología, Fac. Cs. Nat. \& Oceanográficas, U. de Concepción, e-mail: cmurillo@ gmail.com \\ ${ }^{2}$ Depto. de Oceanografía, U. de Concepción, Casilla 160-C, Concepción, Chile, e-mail: coyarzun@udec.cl
}

\section{RESUMEN}

Se estudió la dieta de Dissostichus eleginoides, en tres áreas de la costa centro-sur de Chile, entre $36^{\circ}$ y $51^{\circ} \mathrm{S}$. El análisis de 1583 estómagos, de los cuales el 12,8\% presentaron contenido, indicó que su espectro trófico, alcanzó 22 familias y 26 especies animales. Según el IIR, los peces óseos constituyen el 95,6\% de la dieta, siendo las familias Macrouridae y Ophidiidae las más importantes que pudieron ser identificadas, representando el $21,1 \%$ y $11,2 \%$ del IIR respectivamente, con menor importancia aparecen cefalópodos y sólo ocasionalmente Anthozoa y Polychaeta. No se observa variación intraespecífica en la dieta, asociada al sexo o al tamaño de los individuos, en el rango de tamaños estudiados (57 a $161 \mathrm{~cm}$ de LT). Se observan diferencias geográficas significativas entre las dietas de peces cuyo origen fue el área más austral, respecto de los peces capturados en las áreas septentrionales, en tanto que entre las últimas, la composición de su dieta es similar. Lo anterior se explica por el alto consumo de cefalópodos de la familia Onychoteuthidae en la zona más austral y la nula depredación sobre esta familia en aguas profundas más al norte. Se evidencia variación estacional en la dieta, en las tres áreas estudiadas, así en Primavera-Verano se alimenta preferentemente de peces ofididos, mientras que en la época de OtoñoInvierno, aumenta notoriamente la participación de cefalópodos y peces macrúridos en su dieta, convirtiéndose en todos los casos la última categoría en presa principal. En la época de Otoño-Invierno además, se incrementa el aporte en número de individuos, frecuencia y cantidad de especies de cefalópodos en la dieta de D. eleginoides en las tres áreas analizadas y en particular en el área más austral, en dónde la familia Onychoteuthidae duplica su representación, llegando a más de un $8 \%$ IIR. De acuerdo a los índices H' y J' obtenidos, la especie presentaría una estrecha heterogeneidad trófica, correspondiendo a una especie estenófaga $\left(H^{\prime}=0,50\right)$, lo cual es asociado a una baja uniformidad $\left(\mathrm{J}^{\prime}=0,39\right)$, comportándose como un carnívoro secundario y/o terciario, que depreda principalmente sobre peces Gadiformes y Ophidiiformes demersales, además de cefalópodos téutidos mesopelágicos, alcanzando el último grupo especial importancia en la zona sur de Chile.

Palabras claves: Bacalao de profundidad, ecología trófica, ambientes profundos, talud continental, Chile.

\section{ABSTRACT}

The diet of Dissostichus eleginoides, in three areas of the center-south coast of Chile, between $36^{\circ}$ and $51^{\circ} \mathrm{S}$ was studied. The analysis of 1583 stomachs, of which 12,8\% presented remains, shows a trophic assemblage with 22 families and 26 species. According to the IIR, the bony fish remains constitute 95,6\% of the diet, being Macrouridae and Ophidiidae the most important families, representing $21,1 \%$ and $11,2 \%$ of the IIR respectively with less importance cephalopods and occasionally Anthozoa and Polychaeta also appear. Intraspecific variation in the diet, associated with sex or the size of the individuals was not observed. Significative geographic differences among the diets were observed specially in fish from the most austral area, respect to the fish captured in the most northern areas, whereas the composition of its diet is similar. The previous finding is explained by the high consumption of Onychoteuthidae cephalopods in the most austral zone and the 
null depredation on this item by the northern fish. Seasonal variation in the diet is demonstrated for all studied areas, in Spring-Summer the Patagonian toothfish fed mainly on ophidiid fishes, whereas at the time of Autumn-Winter, increases the participation of cephalopods and rattail fish in its diet, becoming this one the main prey. Also in Autumn-Winter, it is increased the contribution in specimens number, frequency and number of species of cephalopods in the diet of $D$. eleginoides in all areas and specially in the southern most area where the family Onychoteuthidae duplicates its representation, reaching more than $8 \%$ IIR. According to the $\mathrm{H}^{\prime}$ and J' indices, the species displays a narrow trophic heterogeneity, corresponding to a stenophagic species $\left(H^{\prime}=0,50\right)$, which is associated to a low uniformity $\left(J^{\prime}=0,39\right)$, behaving like a secondary and/or tertiary carnivore, that predates mainly on demersal fish like Gadiformes and Ophidiiformes, in addition to mesopelagic teutid cephalopods, reaching the last special group importance in the South zone of Chile.

Keywords: Patagonian Toothfish, trophic ecology, deep sea, continental slope, Chile

\section{INTRODUCCION}

El "bacalao de profundidad", Dissostichus eleginoides Smitt 1898, en su historia de vida realiza una migración ontogenética hacia ambientes profundos, donde es posible encontrarlo sobre la plataforma continental de Sudamérica e islas subantárticas hasta un tamaño de $40 \mathrm{~cm}$ de longitud total (LT), después de lo cual accede a mayores profundidades en el talud continental a medida que crece, variando su alimentación estacional y ontogenéticamente (Arkhipkin et al. 2003). Es capaz de realizar extensas migraciones geográficas, siendo encontrado en lugares tan distantes como la Antártica y Groenlandia (Fischer \& Hureau 1985; Moller et al. 2003). Por su distribución geográfica y su capacidad migratoria, su dieta varía según las características de los ambientes en que se encuentra. D. eleginoides, en su etapa adulta vive en el hábitat profundo, por lo cual el estudio de sus relaciones tróficas es particularmente importante. En Chile, habita en el talud continental y su distribución batimétrica es inversamente proporcional a la latitud, encontrándose cerca del nivel de la plataforma en el sur y a cerca de 2000 metros en el norte. La dieta, del "bacalao de profundidad" en Chile, ha sido parcialmente estudiada, observándose que es un carnívoro ictiófago, teutófago y carcinófago, variando la importancia de estas categorías presa según el estudio consultado (Movillo \& Bahamonde 1971; Flores \& Rojas 1987; Arrizaga et al. 1996). Por otra parte Arrizaga et al. (1996), observan dos grupos tróficos, caracterizados por el consumo de peces óseos $(\leq 90 \mathrm{~cm})$ y escuálidos, canibalismo y cefalópodos $(>90 \mathrm{~cm})$. Con el objeto de aportar información sobre el rol trófico de la especie en esta área de su distribución geográfica, se analizan y presentan los resultados de la dieta de $D$. eleginoides, en el área austral del Pacífico sur oriental y se compara con los resultados de estudios en otras áreas de su distribución.

\section{MATERIALES Y METODOS}

Los peces fueron capturados por pesca artesanal, entre octubre de 2001 y octubre de 2002, y provienen del ambiente profundo del talud continental de Chile, a profundidades superiores a $\operatorname{los} 500 \mathrm{~m}$, con referencias geográficas extremas entre $\operatorname{los} 37^{\circ}$ y $51^{\circ}$ S. Como puertos de desembarque y puntos de comparación, se consideró a Quellón (A1), Valdivia (A2) y Lebu (A3). La pesca de los especimenes se realizó mediante espinel horizontal y la carnada utilizada correspondió a "sardina" y musculatura de "jurel”. El estudio se realizó sobre los estómagos de 1583 ejemplares, los cuales fueron sexados, pesados (g) y medidos en su longitud total LT (cm). Los ítemes presa fueron identificados hasta el nivel taxonómico más bajo posible, sin embargo, frecuentemente solo fue posible reconocerlos hasta el nivel de familia, debido a su avanzado estado de digestión. Los restos no identificados fueron agrupados en categorías más amplias. Cephalopoda y Osteichthyes indeterminados agrupa a moluscos y peces que no fue posible identificar hasta una categoría inferior. Los ítemes-presa con una categoría taxonómica igual o superior a familia y que aparecieron en los contenidos estomacales sólo una vez por área, fueron agrupados en el ítem artificial "Miscelánea". La cuantificación de los contenidos es realizada por los métodos numérico $(\% \mathrm{~N})$ y de frecuencia $(\% \mathrm{~F})$ (Hynes 1950), siendo integrados en el índice de importancia relativa (\%IIR) (Windell 1971). Con los valores del IIR, se calcularon índices de diversidad de ShannonWiener (H') y equidad de Pielou (J') (Calliet et al. 1986). Los datos referentes a sexos, grupos de talla, estaciones y áreas geográficas son comparados estadísticamente usando el coeficiente de correlación por rangos de Spearman (Fritz 1974). Se aplicó análisis de varianza, para evaluar si el tamaño promedio de los peces difiere significativamente entre las áreas de estudio (Zar 1996). Al encontrarse 
diferencias, se aplicó la prueba a posteriori de comparación múltiple tipo Tukey para determinar el o las áreas responsables de las diferencias.

\section{RESULTADOS}

DieTA GENERAL

El rango de tallas de los peces con contenido estomacal varió entre 57 y $161 \mathrm{~cm}$ (promedio = 90,7 cm LT), siendo los ejemplares provenientes de A1 (promedio $\mathrm{LT}=98,5 \pm 18,7 ; \mathrm{n}=83)$ significativamente $\left(\mathrm{F}_{(2,200)}=\right.$ 12,0392; $\mathrm{p}=0,00001$; Test Tukey $\mathrm{p}<0,001)$ de mayor tamaño promedio que los de A2 (promedio LT = $82,7 \pm 21,8 ; \mathrm{n}=49$ ) y A3 (promedio LT $=87,0 \pm 18,9 ; \mathrm{n}=$ 71), los que no difieren entre sí. Se observa un bajo porcentaje de estómagos con contenido estomacal $(12,8 \%)$, lo cual es asociado al método de captura (long-line). En el mismo sentido se observa un alto porcentaje de restos óseos indeterminados $(62,2 \%)$, con los cuales no fue posible reconocer una categoría inferior a Clase. No obstante lo anterior la mayor parte de éstos, probablemente corresponden a las familias Macrouridae y Ophidiidae. El espectro trófico de D. eleginoides, en las áreas de estudio incluye 22 familias que se desglosan en 26 especies animales
(Tabla I). No se observa variación intraespecífica en la dieta de D. eleginoides, asociada al sexo o al tamaño de los individuos en el área de estudio, en el rango de tamaños estudiados (57 a $161 \mathrm{~cm}$ de LT) (ver detalles estadísticos en Murillo 2005). En términos numéricos y de frecuencia, los peces óseos indeterminados (> 32\%) fueron los que más contribuyeron a la dieta de esta especie, seguidos de Macrouridae (> 18\%) y Ophidiidae (>13\%) (Tabla II). Los ítemes alimentarios que presentaron la mayor importancia relativa (IIR) en la dieta del "bacalao de profundidad", en ambientes profundos de la costa centro-sur de Chile fueron: peces óseos indeterminados (IIR\% = $62,2 \%$ ), Macrouridae (IIR\% $=21,1 \%$ ), Ophidiidae (IIR\% $=11,2 \%)$ y Onychoteuthidae $($ IIR $\%=1,3 \%)$ (Tabla II). De acuerdo a los índices H' y J' obtenidos, la especie presentaría una estrecha heterogeneidad trófica, correspondiendo a una especie estenófaga $\left(\mathrm{H}^{\prime}=0,50\right)$, asociada a una baja uniformidad $\left(\mathrm{J}^{\prime}=0,39\right)$, como consecuencia de la gran dominancia de la categoría dietaria peces óseos indeterminados. No obstante se debe considerar que el análisis cuantitativo de la dieta de la especie se realizó sobre la base de información a un nivel taxonómico de familia, por lo que los valores de diversidad y equidad se encuentran relacionados con este aspecto.

Tabla I: Composición taxonómica de los ítemes-presa presentes en los contenidos estomacales de D. eleginoides, durante el período de muestreo octubre 2001-octubre 2002, para las tres áreas estudiadas. e.i: especie indeterminatae. (*): Composición taxonómica, por área geográfica, de los animales encontrados en los contenidos estomacales de D. eleginoides, con los cuales se formó el grupo artificial "Miscelánea" (1= presencia, $0=$ ausencia).

TABLE I: Composition of taxonomic items present in stomach of D. eleginoides, during the sampling period October 2001-October 2002, for the three areas studied. E.I: indeterminatae species. (*): Taxonomic composition, by geographic area, for animals found in stomach contents of $D$. eleginoides, with the artificial group "Miscellany" was fromed $(1=$ presence, $0=$ absence).

\begin{tabular}{|c|c|c|c|c|c|c|}
\hline Clase & Orden & Familia & Especie & $\begin{array}{c}\mathrm{A} 1 \\
\mathrm{n}=83\end{array}$ & $\begin{array}{c}\mathrm{A} 2 \\
\mathrm{n}=49\end{array}$ & $\begin{array}{c}\text { A3 } \\
n=71\end{array}$ \\
\hline Anthozoa * & & & e.i. & 1 & 0 & 0 \\
\hline Polychaeta * & & & e.i. & 0 & 1 & 0 \\
\hline \multirow[t]{11}{*}{ Cephalopoda } & \multirow[t]{8}{*}{ Teuthida } & Ommastrephidae & Dosidicus gigas (D' Orbigny, 1835) & 0 & 1 & 1 \\
\hline & & Ommastrephidae & Sthenoteuthis oualaniensis (Lesson, 1830) & 1 & 1 & 0 \\
\hline & & Onychoteuthidae & Moroteuthis ingens (Smith, 1881) & 1 & 0 & 0 \\
\hline & & Onychoteuthidae & Kondakovia longimana Filippova, 1972 & 1 & 0 & 0 \\
\hline & & Cranchiidae & Cranchia Leach, 1817 & 1 & 1 & 1 \\
\hline & & Cranchiidae & Galiteuthis Joubin, 1898 & 1 & 0 & 0 \\
\hline & & Histioteuthidae & Histioteuthis D’ Orbigny, 1841 & 1 & 0 & 1 \\
\hline & & Octopoteuthidae & Octopoteuthis Rüppell, 1844 & 1 & 1 & 0 \\
\hline & \multirow[t]{3}{*}{ Octopodida } & Opistoteuthidae & Opisthoteuthis Verril, 1883 & 1 & 0 & 0 \\
\hline & & Octopodidae & Pareledone turqueti (Joubin, 1905) & 1 & 0 & 0 \\
\hline & & Octopodidae & Graneledone Joubin, 1918 & 0 & 0 & 1 \\
\hline
\end{tabular}


Dieta de Dissostichus eleginoides en Chile: Murillo, C. ET AL

Continuación TABLA I

\begin{tabular}{|c|c|c|c|c|c|c|}
\hline Crustacea & Decapoda & $\begin{array}{l}\text { Pandalidae } \\
\text { Lithodidae }\end{array}$ & $\begin{array}{l}\text { Heterocarpus reedi Bahamonde, } 1957 \\
\text { Lithodes santolla (Molina, 1782) }\end{array}$ & $\begin{array}{l}0 \\
0\end{array}$ & $\begin{array}{l}0 \\
1\end{array}$ & $\begin{array}{l}1 \\
0\end{array}$ \\
\hline Chondrichthyes & Carcharhiniformes & Scyliorhinidae & Halaelurus canescens (Günther, 1878) & 1 & 1 & 1 \\
\hline \multirow{10}{*}{ Osteichthyes } & Anguilliformes & Congridae * & $e . i$ & 0 & 0 & 1 \\
\hline & Osmeriformes & Alepocephalidae * & $e . i$ & 1 & 0 & 0 \\
\hline & Stomiiformes & Gonostomatidae * & $e . i$ & 1 & 0 & 0 \\
\hline & Ophidiiformes & Ophidiidae & Genypterus maculatus (Tschudi, 1846) & 1 & 1 & 1 \\
\hline & Gadiformes & Macrouridae & Macrourus carinatus (Günther, 1878) & 1 & 1 & 1 \\
\hline & & Macruronidae & Macruronus novaezelandiae Lönberg, 1907 & 1 & 1 & 0 \\
\hline & & Merlucciidae & Merluccius gayi (Guichenot, 1848) & 1 & 1 & 0 \\
\hline & & Moridae* & Antimora rostrata (Günther, 1878) & 1 & 0 & 0 \\
\hline & Perciformes & Nototheniidae & Dissostichus eleginoides Smitt, 1898 & 1 & 0 & 1 \\
\hline & & Carangidae & Trachurus symmetricus (Ayres, 1855) & 0 & 1 & 1 \\
\hline Total especies & & & & 19 & 12 & 11 \\
\hline
\end{tabular}

VARIACIÓN GEOGRÁFICA

El coeficiente de correlación de Spearman $\left(\mathrm{r}_{\mathrm{s}}\right)$, indica que el orden en que son consumidas las presas en la zona más austral es significativamente distinto $(\mathrm{P}>0,05)$ de aquellos provenientes de A2 $\left(\mathrm{r}_{\mathrm{s}}: 0,32\right.$; $\mathrm{P}=0,18)$ y $\mathrm{A} 3\left(\mathrm{r}_{\mathrm{s}}: 0,19 ; \mathrm{P}=0,44\right)$. En tanto que para estas últimas, la composición de su dieta es similar $\left(r_{s}: 0,51 ; P=0,024\right)$. Lo anterior se explica por el alto consumo de cefalópodos de la familia Onychoteuthidae en la zona más austral y la nula depredación sobre esta familia en aguas profundas más al norte (Tabla II). Si bien es cierto, los peces capturados en A1 son más grandes que los procedentes de A2 y A3, el mayor consumo de cefalópodos en la zona más austral, se presenta en los peces de menor tamaño $(\leq 90 \mathrm{~cm} \mathrm{LT})$.

TABLA II. Composición porcentual por área geográfica de los ítemes alimentarios en la dieta de D. eleginoides, expresada a través de los métodos numérico $(\% \mathrm{~N})$, frecuencia de aparición $(\% \mathrm{~F})$ e índice de importancia relativa (\% IIR). Totales: valores sobre los cuales se realizan los cálculos. (*) Miscelánea: Ver Tabla I.|

TABLE II. Percentage composition by geographic area of food items in diet of $D$. eleginoides, expressed by numerical methods $(\% \mathrm{~N})$, and frequency $(\mathrm{F} \%)$ methods and index of relative importance (IIR\%). Totals: values on which the calculations were performed. (*) Miscellaneous: See Table I.

\begin{tabular}{lcccccccccccc}
\hline & & $\mathrm{A} 1$ & \multicolumn{3}{c}{$\mathrm{A} 2$} & & \multicolumn{3}{c}{$\mathrm{A} 3$} & \multicolumn{3}{c}{ Total } \\
\hline & $\% \mathrm{~N}$ & $\% \mathrm{~F}$ & $\% \mathrm{IIR}$ & $\% \mathrm{~N}$ & $\% \mathrm{~F}$ & $\% \mathrm{IIR}$ & $\% \mathrm{~N}$ & $\% \mathrm{~F}$ & $\% \mathrm{IIR}$ & $\% \mathrm{~N}$ & $\% \mathrm{~F}$ & $\% \mathrm{IIR}$ \\
\hline * Miscelánea & 3,9 & 4,8 & 1,0 & 1,9 & 2,0 & 0,2 & 1,3 & 1,4 & 0,1 & 2,6 & 3,0 & 0,4 \\
Ommastrephidae & 1,0 & 1,2 & 0,1 & 3,7 & 2,0 & 0,4 & 1,3 & 1,4 & 0,1 & 1,7 & 1,5 & 0,1 \\
Onychoteuthidae & 10,8 & 13,3 & 7,4 & & & & & & & 4,7 & 5,4 & 1,3 \\
Cranchiidae & 3,9 & 3,6 & 0,7 & 1,9 & 2,0 & 0,2 & 1,3 & 1,4 & 0,1 & 2,6 & 2,5 & 0,3 \\
Histioteuthidae & 2,0 & 2,4 & 0,2 & & & & 2,6 & 2,8 & 0,3 & 1,7 & 2,0 & 0,4 \\
Octopoteuthidae & 2,9 & 2,4 & 0,4 & 1,9 & 2,0 & 0,2 & & & & 1,7 & 1,5 & 0,1 \\
Opistoteuthidae & 2,0 & 2,4 & 0,2 & & & & & & & 0,9 & 1,0 & 0,04 \\
Octopodidae & & & & & & & 2,6 & 2,8 & 0,3 & 0,9 & 1,0 & 0,04 \\
Cephalopoda indet. & 2,9 & 3,6 & 0,6 & & & & 6,5 & 7,0 & 2,1 & 3,4 & 3,9 & 0,7 \\
Crustacea & & & & 3,7 & 4,1 & 0,8 & 5,2 & 5,6 & 1,4 & 2,6 & 3,0 & 0,4 \\
Chondrichthyes & 2,0 & 2,4 & 0,2 & 5,6 & 6,1 & 1,7 & 3,9 & 4,2 & 0,8 & 3,4 & 3,9 & 0,7 \\
Merlucciidae & 2,0 & 2,4 & 0,2 & 1,8 & 2,0 & 0,2 & & & & 1,3 & 1,5 & 0,1 \\
Macruronidae & 3,9 & 4,8 & 1,0 & 5,6 & 6,1 & 1,7 & & & & 3,0 & 3,4 & 0,5 \\
Macrouridae & 15,7 & 19,3 & 15,6 & 20,4 & 22,4 & 22,9 & 22,1 & 23,9 & 24,6 & 18,9 & 21,7 & 21,1 \\
Ophidiidae & 10,8 & 13,2 & 7,4 & 16,7 & 18,4 & 15,3 & 15,6 & 16,9 & 12,3 & 13,7 & 15,8 & 11,2 \\
Carangidae & & & & 5,6 & 6,1 & 1,7 & 2,6 & 2,8 & 0,3 & 2,1 & 2,5 & 0,3 \\
Nototheniidae & 2,9 & 3,6 & 0,6 & & & & 1,3 & 1,4 & 0,1 & 1,7 & 2,0 & 0,2 \\
Osteichthyes indet. & 32,4 & 38,6 & 64,4 & 31,5 & 34,7 & 54,7 & 33,8 & 36,6 & 57,5 & 32,6 & 36,9 & 62,2 \\
Total(100\%) & $\mathrm{n}=102$ & 83,0 & 1937,2 & $\mathrm{n}=54$ & 49,0 & 1995,5 & $\mathrm{n}=77$ & 71,0 & 2149,3 & $\mathrm{n}=233$ & 203 & 1936,4 \\
\hline
\end{tabular}


Gayana 72(1), 2008

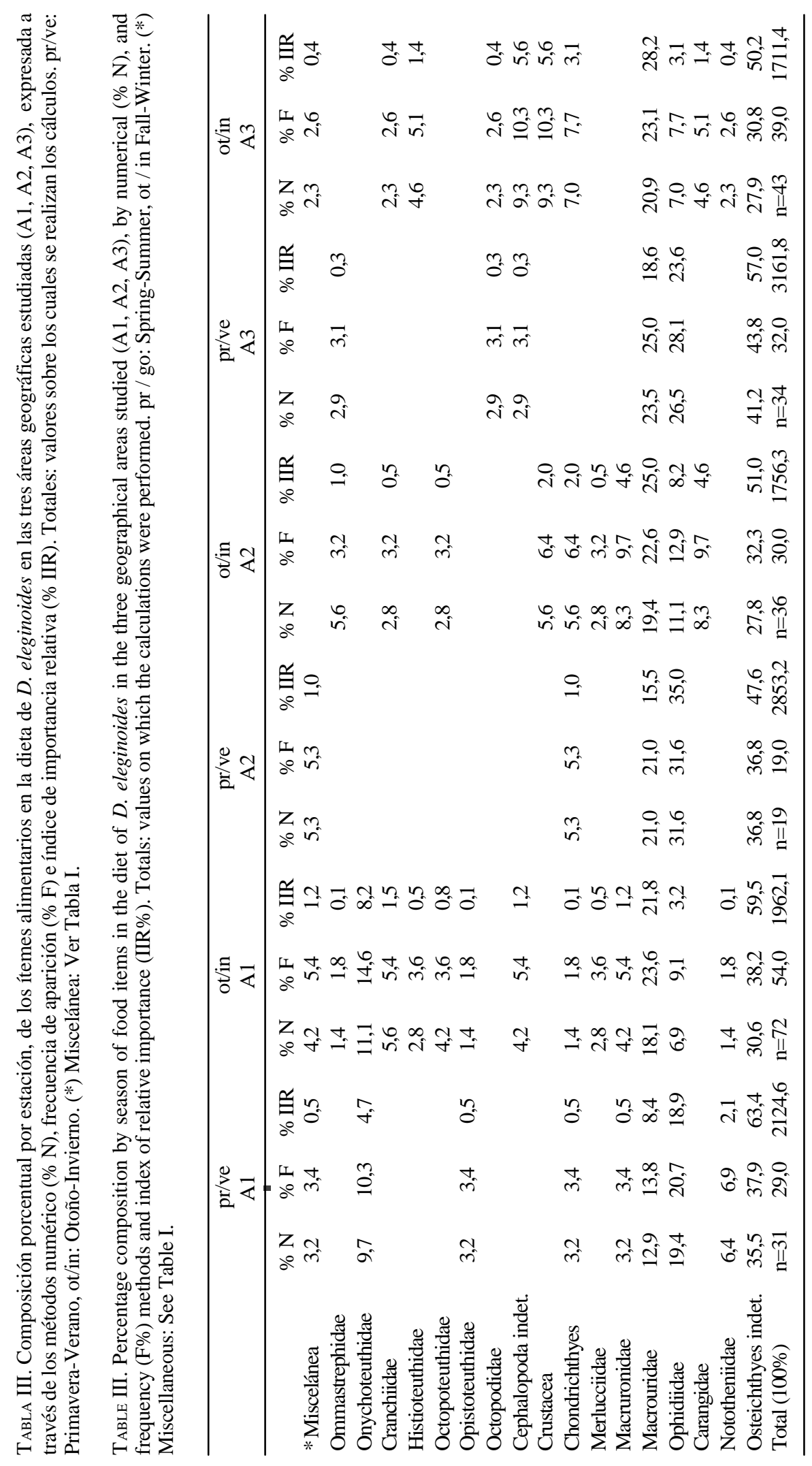


VARIACIÓN ESTACIONAL

Se observa variación estacional en la dieta del "bacalao de profundidad", en las tres áreas estudiadas, en cuanto a la importancia de las categorías identificadas hasta un nivel de familia. Así en Primavera-Verano se alimenta mayormente de peces ofididos, mientras que en la época de Otoño-Invierno, aumenta notoriamente la participación de cefalópodos y peces macrúridos en su dieta, convirtiéndose en todos los casos la última categoría en presa principal (Tabla III). En la época de Otoño-Invierno, se incrementa la importancia de los cefalópodos en la dieta, tanto en número como en frecuencia, lo cual es confirmado por el IIR (\%). También se incrementa el número de familias consumidas, siendo más relevante en el área más austral, dónde la familia Onychoteuthidae duplica su representación, llegando a más de un $8 \%$ IIR en Otoño-Invierno, siendo el aporte total de los cefalópodos superior a 12\% IIR en la misma época, aumentando de dos a seis, el número de familias consumidas entre períodos (Tabla III).

\section{DISCUSION}

DIETA GENERAL

La dieta de $D$. eleginoides en las tres áreas de estudio se compone principalmente de peces óseos y no difiere significativamente entre tamaños ni sexos, lo cual sugiere que en la zona centro-sur de Chile no existiría segregación trófica talla-dependiente, ni sexual, en el rango de tamaños estudiado (57-161 cm de LT). En el mismo sentido Arkhipkin et al. (2003), en un estudio dietario realizado en el área de las islas Malvinas con animales de un amplio rango de tamaños $(16-159 \mathrm{~cm})$, observaron tres grupos tróficos. El primero, se compone de animales menores a $40 \mathrm{~cm}$, que viven sobre la plataforma continental, los cuales son activos depredadores que se alimentan de un amplio espectro de presas de hábitat relacionado con el bentos, tomando una presa a la vez. El segundo grupo trófico, estaría constituido por peces de talla media $(40-60 \mathrm{~cm})$, los cuales se alimentan de presas similares al grupo anterior, pero el número de presas se incrementa de una a dos por pez. El tercer grupo, estaría constituido por individuos grandes, de un tamaño superior a los $60 \mathrm{~cm}$ de LT, los cuales basan su dieta en otros grandes peces demersales, que son consumidos uno a la vez por pez (Macruronus novaezelandiae, Micromesistius australis). Las principales familias de peces-presas del "bacalao de profundidad", capturados en ambientes profundos, en la zona centro-sur de Chile agrupan a especies típicas del talud continental y que poseen amplia distribución a nivel nacional, en particular Ophidiidae y Macrouridae que abundan en el conjunto más profundo de la zona hacia el sur de los $40^{\circ} \mathrm{S}$ (Pequeño 2000).

\section{VARIACIÓN GEOGRÁFICA}

La dieta del "bacalao de profundidad" no difiere significativamente entre las áreas A2 y A3, mientras que entre estas áreas y A1 se presentan diferencias significativas en el consumo de presas. Los crustáceos se encuentran pobremente representados en la dieta de D. eleginoides, encontrándose ausentes en A1. Lo anterior contrasta con otros resultados, en donde esta categoría aportó entre un $17 \%$ y $53 \%$ de la dieta de la especie (Movillo \& Bahamonde 1971; Flores \& Rojas 1987; Arrizaga et al .1996), posiblemente explicado por la mayor abundancia de Heterocarpus reedi en la costa central de Chile. Es notable la presencia de cefalópodos en la dieta de "bacalao de profundidad" capturado en la zona sur de Chile (A1), la cual difiere significativamente de la dieta de peces capturados más al norte del país. Este comportamiento teutófago ha sido también observado en la isla Geogia del Sur (Pilling et al. 2001; Xavier et al. 2002), siendo común para la dieta en estas islas y el sur de Chile, el alto consumo de cefalópodos de la familia Onychoteuthidae. Parece extraño el consumo de "pulpos" del género Octopus(frecuencia de aparición =7\%), indicado por Flores \& Rojas (1987), considerando la distribución batimétrica reportada para las especies de este género en Chile, la cual no supera los 150 metros (Vega et al., 2001). Lo anterior se debería posiblemente a la confusión en la identificación de cefalópodos a partir de restos comunes como mandíbulas. Probablemente, muchos de los ejemplares examinados corresponderían a "calamares" pelágicos y no a "pulpos" bentónicos. En cuanto a antecedentes respecto a la dieta del "bacalao de profundidad", en otras zonas de su distribución geográfica, se ha indicado que en el Atlántico suroccidental, en la plataforma continental de Argentina, el "bacalao de profundidad" con longitudes entre 30 y $95 \mathrm{~cm}$, se alimentaría principalmente de peces nototénidos, siendo también los crustáceos y cefalópodos, grupos presa importante para la especie en esta zona (García de la Rosa et al. 1997). En la isla Georgia del Sur, la dieta de D. eleginoides se encuentra constituida casi en un $50 \%$ de frecuencia, por crustáceos decápodos, isópodos y anfípodos; destacando en menor medida los peces óseos $(\approx 35 \%)$ y los cefalópodos $(\approx$ $10 \%$ ) (Pilling et a., 2001). Por otra parte Xavier et al. (2002), indican que en la misma área el cefalópodo más importante en términos de biomasa, en la dieta de $D$. 
eleginoides es Kondakovia longimana (más del $75 \%$ del aporte de los cefalópodos). En el Océano Indico, en el área de las islas Crozet, la familia Onychoteuthidae representa cerca del $25 \%$ de los cefalópodos ingeridos por D. eleginoides, mientras que en la isla Macquarie la dieta del "bacalao de profundidad" incluye $H$. eltaninae, Chiroteuthis sp., Brachioteuthis picta y Gonatus antarcticus (Hoff 2001). La importancia de los cefalópodos en el área de la isla Macquarie alcanza el $32 \%$ de la biomasa consumida por D. eleginoides, siendo superada sólo por peces, que constituyeron el $58 \%$ de la dieta en términos de biomasa (Goldsworthy et al. 2002). Considerando lo anterior, todos los antecedentes indican variabilidad geográfica en la alimentación del "bacalao de profundidad". En efecto, la misma población de peces cambiaría sus hábitos alimentarios al trasladarse geográficamente con motivo de extensas migraciones, en que sin tener vejiga gaseosa, ocupa la columna de agua, siendo capaz de alimentarse de otros organismos carnívoros tan veloces como "merluzas", "jureles" y "calamares". Lo anterior supone movimientos verticales y horizontales, para lo cual ha desarrollado notables adaptaciones morfológicas y funcionales para facilitar la elevación estática con una flotabilidad neutra, como depósitos de lípidos tegumentarios, escasa mineralización de vértebras, osificación incompleta del neurocráneo e importantes formaciones cartilaginosas en el pedúnculo caudal (Oyarzún et al .1988). La característica migratoria del "bacalao de profundidad" y su capacidad de realizar movimientos verticales, con mínimo gasto energético, lo ha llevado a colonizar nuevos ambientes y ha llegado incluso a superar barreras como la Corriente Circumpolar Antártica y el trópico, reportándose la captura de la especie, en lugares tan insospechados como la Antártica (Fischer \& Hureau 1985) y Groenlandia (Moller et al. 2003), suponiendo este último hallazgo una migración transecuatorial.

\section{VARIACión ESTACIONAL}

Tanto para A1, A2 y A3, se verifica que la dieta del "bacalao de profundidad" en el período PrimaveraVerano fue distinta a la del período Otoño-Invierno (Tabla III). En tal sentido y como característica común a las tres áreas, en el primer período, Ophidiidae es el ítem-presa que presenta un mayor aporte en términos de \%IIR a la dieta, mientras que en Otoño-Invierno, la representación de este grupo disminuye, aumentando notoriamente la representación de Macrouridae. Otro aspecto importante es el incremento en el aporte en número, frecuencia y especies de cefalópodos en la dieta de D. eleginoides en las tres áreas y en particular para el caso de la zona más austral, en dónde la familia Onychoteuthidae duplica su representación, llegando a más de un $8 \%$ IIR en Otoño-Invierno, siendo el aporte total de los cefalópodos superior a 12\% IIR en el mismo período, aumentando de dos (Onychoteuthidae y Opistoteuthidae)aseis(Ommastrephidae, Onychoteuthidae, Cranchiidae, Histioteuthidae, Octopoteuthidae y Opistoteuthidae), el número de familias consumidas entre períodos. En A2, no se registró consumo de cefalópodos en el primer período, observándose la depredación sobre 3 familias en Otoño-Invierno (Ommastrephidae, Cranchiidae y Octopoteuthidae), aunque con un aporte total cercano al 2\% IIR. Para el caso de A3, el número de familias consumidas aumenta de dos (Ommastrephidae y Octopodidae), con un 0,9\% IIR a tres (Cranchiidae, Histioteuthidae y Octopodidae) con un 7,7\% IIR. El aparente aumento en el consumo de cefalópodos en el período de Otoño-Invierno, puede relacionarse con la oportunidad de encuentro entre el depredador y sus presas, en el período de migración de D. eleginoides y de especies de cefalópodos. En efecto, se ha reportado la migración de la principales especies de cefalópodos presa del "bacalao de profundidad" con fines reproductivos en la época invernal. Así, Cherel \& Weimerskirch (1999), indican que los onicotéutidos $K$. longimana y $M$. ingens, presentes en las islas Crozet, desovan en los meses de Invierno. En otras áreas, como islas Malvinas y Nueva Zelanda se confirma el desove de Invierno de $M$. ingens, cuyas hembras realizan migraciones desde la plataforma continental hacia aguas más profundas para desovar en esta estación del año (Jackson 2001). Otro aspecto importante, relacionado con la oferta de onicotéutidos, corresponde a que la máxima biomasa de $M$. ingens, al menos en el sur de Nueva Zelanda ocurre a una profundidad cercana a los 700 metros, manteniendo alta abundancia hasta los 1200 metros de profundidad (Jackson et al. 2000). Por otra parte, Arkhipkin et al. (2003), observan variación estacional en la dieta de peces de talla media (40-60 cm LT), que se encuentran sobre la plataforma continental de Argentina, la cual se encuentra relacionada con la abundancia y migraciones de sus especies presa principales, particularmente del calamar Loligo gahi, que se encuentra en los contenidos estomacales en Otoño-Invierno, cuando realiza una migración estacional costa afuera hasta el rango batimétrico de $D$. eleginoides. Posteriormente, en Primavera-Verano, L. gahi desaparece de la dieta debido a su migración reproductiva cerca a la costa.

Eastman (1991), indica que entre los nototénidos se 
distinguen "varios tipos ecológicos", los que en aguas templadas normalmente son ocupados por peces de diferentes categorías taxonómicas, indicando que $D$. eleginoides corresponde probablemente al depredador mesopelágico de mayor tamaño. En efecto, el "bacalao de profundidad", se presenta como un depredador de alto nivel trófico (carnívoro secundario y/o terciario), que es capaz de ascender en la columna con un mínimo gasto energético y alimentarse de organismos que habitan este ambiente, presentando variación estacional y geográfica en su dieta, de acuerdo a la disponibilidad de presas en un período del año o del área geográfica que habita.

\section{BIBLIOGRAFIA}

ArkHIPKIN, A., P.B RICKLE \& V. LAPTIKHOVSKY. 2003. Variation in the diet of the Patagonian toothfish with size, depth and season around the Falkland Islands. Journal of Fish Biology, 63(2): 428-441.

Arrizaga, A., M. Fuentealba, V. Yáñez \& C. Oyarzún. 1996. Observaciones sobre la alimentación de Dissostichus eleginoides Smitt, 1898 (Perciformes, Nototheniidae) en el sur de Chile. Gayana Oceanología (Chile), 4(1): 13-19.

Calliet, G., M. Love \& A. Ebeling. 1986. Fishes a field and laboratory manual on their structure, identification and natural history. Second Edition. Wadsworth Publishing Company Belmont, California, 194 pp.

Cherel, Y. \& H. Weimerskirch. 1999. Spawning cycle of onychoteuthid squids in the southern Indian Ocean: new information from seabird predators. Marine Ecology Progress Series, 188: 93-104.

EAstman, J. 1991. Evolution and Diversification of Antarctic Notothenioid Fishes. American Zoologist, 31:93-109.

FisCHER, W. \& J. HUREAU. 1985. FAO species identification sheets for fishery purposes. Southern Ocean (Fishing Areas 48, 58 and 88) (CCAMLR Convention Area). Prepared and published with the support of the Comission for the Conservation of Antarctic Marine Living Resources. Rome. Vol.1-2: 470 pp.

FloRes H. \& P.R OJas. 1987. Contenido gástrico de Dissostichus eleginoides Smitt 1898, capturado frente a Valparaíso (Perciformes - Nototheniidae). Investigaciones Marinas (Chile), 15: 33-40.

Fritz, E. 1974. Total diet comparison in fishes by Spearman rank correlation coefficients. Copeia, 1974 (1): 210-214.

García de la Rosa, S., F. Sánchez\& D. F igueroa. 1997. Comparative feeding ecology of patagonian tootfish (Dissostichus eleginoides) in the Southwestern Atlantic. CCAMLR Science, 4: 105-124.

Goldsworthy, S., M. Lewis, R. Williams, X. He, J. Young \&
J. vAN DEN HofF. 2002. Diet of toothfish ( Dissostichus eleginoides ) around Macquarie Island, South Pacific Ocean. Marine Freshwater Research, 53: 49-57.

Hoff, J. 2001. Further observations on the cephalopod diet of wandering albatrosses (Diomedea axulans) at Macquarie Island. EMU, 101: 169-172.

Hynes, H. 1950. The food of freshwater sticklebacks (Gasterosteus aculeatus and Pygosteus pungitus) with a review of methods used in studies of the food of fishes. Journal of Animal Ecology, 19: 36-58.

$\mathrm{J}_{\mathrm{ACKSON}}$, G. 2001. Confirmation of winter spawning of Moroteuthis ingens (Cephalopoda: Onychoteuthidae) in the Chatham Rise región of New Zealand. Polar Biology, 24: 97-100.

J ACKSON, G., A. Shaw \& C. Lalas. 2000. Distribution and biomass of two squid species off southern New Zealand: Nototodarus sloanii and Moroteuthis ingens. Polar Biology, 23: 699-705.

Moller, P.R., J.G.N IELSEN \& I. Fossen. 2003. Fish migration: Patagonian toothfish found off Greenlaand. Nature, 421: 599.

Movillo, J. \& N. Bahamonde. 1971. Contenido gástrico de Dissostichus amissus Gill y Townsend en San Antonio. Noticiario Mensual Museo Nacional de Historia Natural (Chile), 15(175): 9-11.

Murillo, C.A. 2005. Ecología trófica de Dissostichus eleginoides Smitt, 1898 (Osteichthyes: Nototheniidae) en ambientes profundos de la costa Centro Sur de Chile. Tesis para optar al Grado de Magíster en Ciencias, Mención Zoología. Universidad de Concepción. 78 pp.

Oyarzún, C., P. CAmpos \& H. Valeria. 1988. Adaptaciones para la flotabilidad en Dissostichus eleginoides Smitt, 1898 (Pisces, Perciformes, Nototheniidae). Investigaciones Pesqueras (España), 52(4): 455-466.

Pequeño, G. 2000. Delimitaciones y relaciones biogeográficas de los peces del Pacífico suroriental. Estudios Oceanológicos (Chile), 19: 53-76.

Pilling, G., M. Punves, T.D aw, D. Agnew \& J. Xavier. 2001. The stomach contents of Patagonian toothfish around South Georgia (South Atlantic). Journal of Fish Biology, 59: 1370-1384

Vega, M., S. Letelier \& E. Carreño. 2001. Colección de cefalópodos del Museo Nacional de Historia Natural: Catálogo de especies de aguas chilenas. Publicación Ocasional del Museo Nacional de Historia Natural (Chile), 57: 5-86.

WindELL, J. 1971. Food analysis and rate of digestion. En: Ricker W.E. Editor. Methods for assessment of fish production in fresh waters. Second edition, IBPHandbook $\mathrm{N}^{\circ} 3$, Pags. 215-226.

Xavier, J., P. Rodhouse, M. Purves, T. Daw, J. Arata \& G. PILLING. 2002. Distribution of cephalopods recorded in the diet of the Patagonian toothfish (Dissostichus eleginoides ) around South Georgia. Polar Biology, 25: 323-330.

ZAR, J. 1996. Biostatistical analysis. Third Edition. PrenticeHall Inc. USA. 662 pp. 